\title{
A Transformation Linking Two Models of Coagulation
}

\author{
ROBERT M. ZIFF, ${ }^{1}$ M. H. ERNST, AND E. M. HENDRIKS \\ Department of Chemical Engineering, The University of Michigan, Ann Arbor, Michigan 48109 and \\ Institute for Theoretical Physics, State University of Utrecht, 3508 TA Utrecht, The Netherlands
}

Received July 21, 1983; accepted December 21, 1983

\begin{abstract}
It is shown that a given solution to Smoluchowski's coagulation equation with the kernel $K_{i j}$ $=(a i+b)(a j+b)$ can be directly transformed to obtain a solution to the same equation with $K_{i j}=a(i+j)+b$.
\end{abstract}

The evolution of the distribution of particle size, in systems where coagulation or agglomerization takes place, is described by Smoluchowski's coagulation equation,

$$
d c_{k} / d t=\frac{1}{2} \sum_{i+j=k} K_{i j} c_{i} c_{j}-c_{k} \sum_{j} K_{k j} c_{j}
$$

where $c_{k}(t)$ is the cluster size distribution and $K_{i j}$ are the rate constants of the coagulation process. (Unless otherwise indicated, sums are over all positive integers.) Complete, explicit solutions to [1] have been found only when $K_{i j}$ is given by $1, i+j, i j$, and their linear combinations (1). In this paper, we show that two classes of soluble models, with $K_{i j}=(a i$ $+b)(a j+b)=s_{i} s_{j}($ model I $)$, and $K_{i j}=a i$ $+a j+b=s_{i+j}$ (model II), are closely related, such that a given solution of one model can be directly transformed into a solution of the other $\left(s_{k} \equiv a k+b\right)$. These two models are directly applicable to two systems of condensation polymerization. In the following, we give a derivation of this transformation and apply it to some special examples. We also discuss the generalization to continuum models.

The total mass or number of structural units, $\Sigma k c_{k}(t)$, remains constant, unless ge-

\footnotetext{
'To whom correspondence should be sent.
}

lation (infinite cluster growth) occurs. For those $K_{i j}$ where gelation occurs, mass conservation is valid only up to the onset of gelation, at time $t_{c}$, and the solution past the gel point requires special considerations $(2,3)$. In model I gelation occurs in a finite time (as shown below); however, the post-gel solution will not be of concern here since only the behavior for $t<t_{c}$ is relevant for the transformation.

Let $c_{k}(t)$ be a solution model I, described by the coagulation equation

$$
d c_{k} / d t=\frac{1}{2} \sum_{i+j=k} s_{i} s_{j} c_{i} c_{j}-s_{k} c_{k} \sum_{j} s_{j} c_{j}
$$

for a given initial condition $c_{k}(0)$. To make the transformation we multiply [2] by $s_{k}$ and introduce $\tilde{c}_{k} \equiv s_{k} c_{k}$, with the result

$$
d \tilde{c}_{k} / d t=\frac{1}{2} \sum_{i+j=k} s_{i+j} \tilde{c}_{i} \tilde{c}_{j}-\tilde{c}_{k} s_{k} \sum \tilde{c}_{j}
$$

We can recast the RHS of [3] in the form of the coagulation equation for model II, with $K_{i j}=s_{i+j}$, by writing $s_{k}=s_{k+j}-a j$. We thus find

$$
\begin{aligned}
& \frac{d \tilde{c}_{k}}{d t}-a \tilde{c_{k}} \sum_{j} j \tilde{c}_{j} \\
& \quad=\frac{1}{2} \sum_{i+j=k} s_{i+j} \tilde{c}_{i} \tilde{c}_{j}-\tilde{c}_{k} \sum_{j=1}^{\infty} s_{k+j} \tilde{c}_{j} .
\end{aligned}
$$


Multiplying [2] by $k s_{k}$ and summing over $k$, we find that the quantity $m(t)$, defined by

$$
m(t)=a \sum_{k} k \tilde{c}_{k}(t)=a \sum_{k} k s_{k} c_{k}(t)
$$

satisfies

$$
d m / d t=m^{2}
$$

or

$$
m(t)=\left(t_{c}-t\right)^{-1}
$$

valid for $t<t_{c}$. The point $t_{c}$, where $m$ diverges, marks the onset of gelation for this model. The value of $t_{c}$ is determined by the initial condition as

$$
1 / t_{c}=a \sum_{k} k \tilde{c}_{k}(0)=a \sum_{k} k s_{k} c_{k}(0)
$$

which is a combination of the first and second moments of $c_{k}$.

On the account of [6] the LHS of [4] may be written as $m(d / d t)\left(\tilde{c}_{k} / m\right)$. As the next step we introduce new variables $c_{k}^{*}(\tau)=\tilde{c}_{k}(t) / m(t)$ and $d \tau=m(t) d t$, which allows us to write [4] in the form

$$
d c_{k}^{*} / d \tau=\frac{1}{2} \sum_{i+j=k} s_{i+j} c_{i}^{*} c_{j}^{*}-c_{k}^{*} \sum_{j} s_{k+j} c_{j}^{*}
$$

where $c_{k}^{*}(\tau)$ is seen to satisfy the coagulation equation for model II. Integration of $d \tau=m d t$ with $\tau(0)=0$ yields

and

$$
t=t_{c}\left(1-e^{-\tau}\right)
$$

$$
\begin{aligned}
c_{k}^{*}(\tau) & =\tilde{c}_{k}(t) / m(t) \\
& =t_{c} e^{-\tau} s_{k} c_{k}\left(t_{c}\left(1-e^{-\tau}\right)\right) .
\end{aligned}
$$

The total mass in the new model is given by

$$
\sum_{k} k c c_{k}^{*}(\tau)=1 / a
$$

on account of [9], [10], and [6]. The mass can be made equal to any value of rescaling $\tau$ and $c_{k}^{*}(\tau)$. Following standard convention, we choose the mass to be unity, and thus rescale as

$$
n_{k}(\tau) \equiv a c_{k}^{*}(a \tau) .
$$

This completes the transformation.
In summary, to transform a solution $c_{k}(t)$ of model I to a solution $n_{k}(t)$ of model II, we have found

$$
n_{k}(t)=a(a k+b) t_{c} e^{-a t} c_{k}\left(t_{c}\left(1-e^{-a t}\right)\right) .
$$

The initial conditions of these two solutions are related by

$$
n_{k}(0)=a(a k+b) t_{c} c_{k}(0)
$$

and the mass in model II is normalized to unity:

$$
\sum_{k} k n_{k}(t)=1 .
$$

It follows, especially, that a solution for a monodisperse initial condition in one model is transformed into a solution for a monodisperse initial condition of the other. The quantity $t_{c}$ in the above transformation is determined from the initial distribution in model I by [7]. To transform model II to model I we invert [13], to find

$$
c_{k}(t)=\frac{n_{k}\left[-(1 / a) \ln \left(1-t / t_{c}\right)\right]}{a(a k+b)\left(t_{c}-t\right)}
$$

where the initial conditions are still related by [14]. To find $t_{c}$ we set the total mass of system I equal to unity, $\Sigma k c_{k}(0)=1$. Then it follows directly from [14]:

$$
t_{c}=\sum_{k} k n_{k}(0) /[a(a k+b)] .
$$

In model II, $t_{c}$ does not mark any singularity, but only represents a parameter of the transformation based upon the initial behavior of $n_{k}(0)$. The time interval $0<t<t_{c}$ in model I is mapped to the interval $0<t<\infty$ in model II, so that the pre-gel solution of the former is stretched to a complete solution to latter, with the singular (gel) point moved to $t=\infty$. In the reverse transformation, the entire solution of model II is compressed to the pregel solution of model I.

Now we consider some examples and applications. The polymerization of $f$-functional branched monomers, $R A_{f}$ (where $f$ is an integer larger than unity) is described by the 
coagulation equation with $K_{i j}=[(f-2) i$ $+2] \cdot[(f-2) j+2](4-7)$, and the solution for a monodisperse initial condition, $c_{k}(0)$ $=\delta_{k 1}$, is given by

$c_{k}(t)=\frac{f(f k-k) !}{(f k-2 k+2) !} \frac{(f t)^{k-1}}{k !(1+f t)^{(f-1) k+1}}$

where $t_{c}=1 / f(f-2)$ (which follows from [7]). Applying the transformation with $a=(f$ $-2)$ and $b=2$, we find

$$
\begin{aligned}
n_{k}(t)=\frac{e^{-(f-2) t}(f k-k) !}{(f k-2 k+1) ! k !}\left(\frac{1-e^{-(f-2) t}}{f-2}\right)^{k-1} \\
\quad \times\left(1+\frac{1-e^{-(f-2) t}}{f-2}\right)^{-1-(f-1) k}
\end{aligned}
$$

for the solution with $K_{i j}=(f-2)(i+j)+2$ and monodisperse initial conditions, $n_{k}(0)$ $=\delta_{k 1}$, which describes the polymerization of $A R B_{f-1}$ branched molecules (4-8).

As another example, consider the solution for $K_{i j}=i j$, again for monodisperse initial conditions:

$$
c_{k}(t)=t^{k-1} e^{-k t} k^{k-2} / k !
$$

Applying [13], we find

$$
\begin{aligned}
n_{k}(t)=e^{-t} & \left(1-e^{-t}\right)^{k-1} \\
& \times \exp \left(-k\left(1-e^{-t}\right)\right) k^{k-1} / k !
\end{aligned}
$$

as the solution for $K_{i j}=i+j$ with monodisperse initial conditions.

The transformation may be used to extend to the model $K_{i j}=i+j$ the many known results for the model $i j(2,3)$. For example, as $t \uparrow t_{c}=1 / M_{2}(0)$, the asymptotic behavior of $c_{k}$ for large $k$ is given by (3)

$$
\begin{aligned}
c_{k} \simeq\left(2 \pi \mu t_{\mathrm{c}}^{3}\right)^{-1 / 2} & k^{-5 / 2} \\
& \times \exp \left[-k\left(t-t_{c}\right)^{2} / 2 \mu t_{c}^{3}\right]
\end{aligned}
$$

where $\mu=M_{3}(0)$ and $M_{n}=\Sigma k^{n} c_{k}$, and $M_{1}$ is assumed to be 1 . This is valid in the limit $k \rightarrow \infty, t \uparrow t_{\mathrm{c}}$ with $k\left(t-t_{\mathrm{c}}\right)^{2}$ fixed. Note that [22] depends only upon $M_{1}, M_{2}$, and $M_{3}$ of the initial distribution. Applying [13] and [14], we find

$$
n_{k}(t) \simeq(B / \pi)^{1 / 2} k^{-3 / 2} e^{-t} \exp \left[-B k e^{-2 t}\right]
$$

with

$$
B^{-1}=2 \sum k^{2} n_{k}(0)
$$

for the large $k$-and $t$-behavior of the size distribution in the model $K_{i j}=i+j(9,10)$. This result is valid in the coupled limit of $k \rightarrow \infty$ and $t \rightarrow \infty$ with $k e^{-2 t}$ constant.

The transformation may be extended to the case of continuous variables, where Smoluchowski's equation is written in integral form. Here, because the size variable, $x$, can also be scaled, one can choose units so that two initial moments are arbitrarily given. We assume units are chosen such that $M_{1}(0)=M_{2}(0)$ $=1$. Let the size distribution $c(x, t)$ be the solution for a transition kernel $K(x, y)=x y$ (model $\mathrm{I}$ ) and $n(x, t)$ be a solution for the kernel $K(x, y)=x+y$ (model II). The analog of [13] becomes, for general initial conditions:

$$
n(x, t)=e^{-t} x c\left(x,\left(1-e^{-t}\right)\right) .
$$

Note that $t_{c}=1$ because of the choice $M_{2}$ $=1$. If we apply the transformation [25] to the closed form solution of the model $K(x, y)$ $=x y(11)$ :

$$
c(x, t)=e^{-(1+t) x} I_{1}(2 x \sqrt{t}) /\left(x^{2} \sqrt{t}\right)
$$

corresponding to the initial condition $x c(x, 0)$ $=e^{-x}$, then we find the solution for the model $K(x, y)=x+y(12)$ :

$$
\begin{aligned}
& n(x, t)=x^{-1}\left(1-e^{-t}\right)^{-1 / 2} e^{-t} \\
& \quad \times \exp \left[-x\left(2-e^{-t}\right)\right] I_{1}\left[2 x\left(1-e^{-t}\right)^{1 / 2}\right]
\end{aligned}
$$

corresponding to the initial condition $n(x, 0)$ $=e^{-x}$. The transformation may also be applied to the infinite-series solutions of I and II given in Refs. $(1,13)$.

\section{ACKNOWLEDGMENTS}

R.M.Z., a Shell Faculty Fellow, acknowledges support of the Shell Foundation. M.H.E. and E.M.H. thank the Department of Chemical Engineering of The University of Michigan for its hospitality during their visit. 


\section{REFERENCES}

1. Drake, R. L., in "Topics in Current Aerosol Research" (G. M. Hidy and J. R. Brock, Eds.), Vol. 3, Part 2. Pergamon, Elmsford, N. Y., 1972.

2. Leyvraz, F., and Tschudi, H. R., J. Phys. A: Math. Gen. A 14, 3389 (1981).

3. Ziff, R. M., Ernst, M. H., and Hendriks, E. M., $J$. Phys. A: Math. Gen. 16, 2293 (1983).

4. Ziff, R. M., J. Stat. Phys. 23, 241 (1980).

5. Stockmayer, W. H., J. Chem. Phys. 11, 45 (1943).

6. Flory, P. J., "Principles of Polymer Chemistry." Cornell Univ. Press, Ithaca, N. Y., 1953.
7. Cohen, R. J., and Benedek, G. B., J. Phys. Chem. 86, 3696 (1982).

8. Lushnikov, A. A., J. Colloid Interface Sci. 65, 276 (1978).

9. Lushnikov, A. A., J. Colloid Interface Sci. 48, 400 (1974).

10. Scott, W. T., J. Atm. Sci. 25, 54 (1968).

11. McLeod, J. B., Proc. London Math. Soc. 14, 445 (1964).

12. Golovin, A. M., Izv. Geophys. Ser. 1963, 783 (English Trans: Bull. Acad. Sci. USSR, Geophys. Ser., No. 5, 482 (1963)).

13. Ernst, M. H., Ziff, R. M., and Hendriks, E. M., $J$. Colloid Interface Sci. 97, 266 (1984). 